\title{
IMPLEMENTATION AND TESTING OF A NEW OPENFOAM SOLVER FOR PRESSURE-DRIVEN LIQUID FLOWS ON THE NANOSCALE
}

\author{
ALEXANDROS STAMATIOU, S. KOKOU DADZIE, \& ALWIN M. TOMY \\ School of Engineering and Physical Sciences, Heriot-Watt University, Scotland, UK
}

\begin{abstract}
Over the past two decades, several researchers have presented experimental data from pressure-driven water flow through carbon nanotubes quoting mass flow rates which are four to five orders of magnitude higher than those predicted by the Navier-Stokes equations with no-slip condition. The current work examines the development of an OpenFOAM solver for creeping flows that better accounts for some micro- and nano-scale diffusion processes. It is based on the observation that a change of velocity variable within the classical Navier-Stokes equations leads to a form of flow model with additional diffusive terms which become apparent at the micro- and nano-scale. Numerical simulations from the new solver compare well with associated analytical solutions that match the experimental flow enhancement observed in cylindrical tubes. This lays the foundations for further investigations of liquid flows in more complex nano-sized geometries, such as those obtained from pore-scale imaging.
\end{abstract}

Keywords: micro- and nanofluidics, continuum models, mass/volume diffusion, Navier-Stokes equations.

\section{INTRODUCTION}

Water transport in Carbon nanotubes (CNTs) has been a subject of intense research over the past two decades, predominantly because of its potential applications in technologies such as molecular level drug delivery and nanofiltration. The first major experimental studies of liquid flow through CNT membranes were carried out by Majumder et al. [1] and Holt et al. [2], on $7 \mathrm{~nm}$ diameter and 1.3-2 $\mathrm{nm}$ diameter CNTs respectively. Both investigations suggested extremely large water flow rate enhancements when compared to predictions based on the no-slip Haagen-Poiseuille flow law. In a repeat of the experiment, Majumder et al. [3] were later able to confirm their original findings of enhancement factors on the order of $10^{3}-10^{4}$. In contrast to this, Qin et al. [4] reported flow enhancements of order $10^{2}-10^{3}$ in $0.81-1.59$ $\mathrm{nm}$ diameter CNTs, while the results by Du et al. [5] pointed to an enhancement of up to order $10^{5}$ in $10 \mathrm{~nm}$ diameter channels. With the aim of expanding the data-set to include also wider tube diameters, Whitby et al. [6] investigated liquid flow through CNTs of $44 \mathrm{~nm}$ diameter, calculating only a 20-37 fold enhancement over no-slip Hagen-Poiseuille flow. For 200-300 nm diameter tubes, Sinha et al. [7] found no significant deviation, suggesting that flow enhancement effects diminish with increasing diameter.

A wide variety of flow enhancement data can also be found in the literature on molecular dynamics simulations of water flow through CNTs. For instance, Joseph and Aluru [8] measured an enhancement factor of 2052 in $2.17 \mathrm{~nm}$ diameter channels, while Thomas and McGaughey [9] found enhancements of 144-176 in 1.66-4.99 nm channels. Walther et al. [10] even report enhancements as low as 32, 25, 22 in simulations of 2.71, 4.07, $5.42 \mathrm{~nm}$ diameter tubes. Even if the spread in these data is large, the molecular dynamics simulations are consistent in the sense that none of them confirms the extremely high enhancement values found in some of the experimental studies. In fact, by employing the Young-Laplace equation, Walther et al. [10] study the water entry and filling stages of a CNT and derive a maximum attainable flow enhancement factor of 253. A similar upper bound is also argued 
for by Sisan and Lichter [11] using continuum methods. They show that frictional entrance and exit losses should not be neglected even for channels of small aspect ratio, thus limiting the flow rate.

It is common practice to quantify the flow enhancement using the slip velocity, which is a correction of the no-slip condition by the introduction of a constant velocity at the wall, leading to a slip-modified Haagen-Poiseuille mass flow rate. In experiments, the slip velocity is then found retrospectively by substituting the (enhanced) flow rate into this equation. A great deal of research has gone into effectively predicting the slip velocity a priori for a given fluid-solid combination. Using molecular dynamics simulations, this is often done by analysis of the parabolic velocity profile (see e.g. [9], [12]) or by use of the Navier friction coefficient (see [13], [14]). A different approach was suggested by Myers [15]. Leaving intact the no-slip condition, he modeled the flow enhancement by incorporating a region of reduced viscosity near the wall.

In Stamatiou et al. [16], a novel modelling approach was introduced in which the flow enhancement is caused by a diffusion mechanism that only becomes apparent at the nanoscale. A unifying recasting methodology was proposed by which a new class of continuum models termed Recast Navier-Stokes equations (RNS), can be directly derived from the Navier-Stokes equations [17], [18]. The idea is based on transforming the velocity vector field within the classical equations in a way that depends on the driving mechanism of the flow (for liquid flow in CNTs, this is the pressure gradient). The mass flow rate derived from this model as compared with experimental data showed reasonable agreement [16].

The objective of the current work is to present and validate a numerical implementation of the Recast Navier-Stokes equations in a new OpenFOAM solver (rnsLiquidFoam). For creeping flows in cylindrical tubes of small aspect ratio, a perturbation analysis yielding analytical expressions for the pressure and velocity fields are obtained [16]. These solutions are reviewed and used to validate the solver. The new solver can then be applied to simulate flows in complex geometries such as those obtained from pore-scale imaging techniques.

In Sections 2 and 3, the proposed equations for pressure-driven liquid flows and their numerical implementations are presented. In Sections 4 and 5, the perturbation solutions are reviewed and the numerical solutions are compared against them.

\section{RECAST NAVIER-STOKES EQUATIONS}

For a fluid of constant mass density $(\rho)$, the Navier-Stokes mass and momentum conservation laws may be written as follows:

$$
\begin{gathered}
\nabla \cdot \mathrm{U}_{\mathrm{m}}=0, \\
\frac{\partial}{\partial t}\left(\rho \mathrm{U}_{\mathrm{m}}\right)+\nabla \cdot\left(\rho \mathrm{U}_{\mathrm{m}} \otimes \mathrm{U}_{\mathrm{m}}\right)+\nabla \cdot\left[p \mathbf{I}+\Pi^{(N S)}\right]=0 .
\end{gathered}
$$

Here, $\Pi^{(N S)}$ is the Newtonian stress tensor which is given in terms of the fluid's dynamic viscosity $(\mu)$ as:

$$
\Pi^{(N S)}=-2 \mu\left[\frac{1}{2}\left(\nabla \mathrm{U}_{\mathrm{m}}+\left(\nabla \mathrm{U}_{\mathrm{m}}\right)^{\mathrm{T}}\right)-\frac{1}{3} \mathbf{I}\left(\nabla \cdot \mathrm{U}_{\mathrm{m}}\right)\right]=-2 \mu \frac{\circ}{\nabla \mathrm{U}_{\mathrm{m}}} .
$$

The unknown velocity field $\mathrm{U}_{\mathrm{m}}=\mathrm{U}_{\mathrm{m}}(x, t)$ is that of the conventional mean mass velocity. If an externally applied pressure gradient is the principal driving mechanism of the flow, the new theory presented in Reddy et al. [17] and Dadzie and Reddy [18] assumes that the classical mass velocity can be written in terms of a new pressure diffusion velocity $\mathrm{U}_{\mathrm{p}}$ as:

$$
\mathrm{U}_{\mathrm{m}}=\mathrm{U}_{\mathrm{p}}-\kappa_{\mathrm{p}} \nabla \ln p=\mathrm{U}_{\mathrm{p}}-\kappa_{\mathrm{p}} \frac{\nabla p}{p} .
$$


The second term on the right hand side represents a mass diffusion mechanism driven by the pressure gradient. This distinction between $\mathrm{U}_{\mathrm{m}}$ and $\mathrm{U}_{\mathrm{p}}$ is analogous to the idea of a volume velocity in gas [19]-[21]. The molecular pressure diffusivity coefficient $\kappa_{\mathrm{p}}$ is assumed to be of the form:

$$
\kappa_{p}=\alpha^{*} \frac{\mu}{\rho}=\alpha^{*} \nu
$$

where $\alpha^{*}$ is a dimensionless parameter and $\nu$ the fluid's kinematic viscosity. Substituting eqn (4) into the Navier-Stokes eqns (1)-(2) and re-arranging terms leads to the recast NavierStokes (RNS) equations for mass and momentum:

$$
\begin{aligned}
\nabla \cdot \mathrm{U}_{\mathrm{p}}= & \nabla \cdot\left(\kappa_{\mathrm{p}} \nabla \ln p\right), \\
& \frac{\partial}{\partial t}\left(\rho \mathrm{U}_{\mathrm{p}}-\kappa_{\mathrm{p}} \rho \nabla \ln p\right)+\nabla \cdot\left(\rho \mathrm{U}_{\mathrm{p}} \otimes \mathrm{U}_{\mathrm{p}}\right)=\nabla \cdot \mathbf{T}^{(R N S)},
\end{aligned}
$$

with the tensor $\mathbf{T}^{(R N S)}$ on the right-hand side of this equation given by,

$$
\begin{aligned}
\mathbf{T}^{(R N S)} & =\left(-p-\frac{2}{3} \frac{\mu \kappa_{\mathrm{p}}}{p^{2}}|\nabla p|^{2}+\frac{2}{3} \frac{\mu \kappa_{\mathrm{p}}}{p} \nabla^{2} p\right) \mathbf{I}+\frac{\kappa_{\mathrm{p}}}{p^{2}}\left(2 \mu-\rho \kappa_{\mathrm{p}}\right) \nabla p \otimes \nabla p+2 \mu \mathrm{D}\left(\mathrm{U}_{\mathrm{p}}\right) \\
& -\frac{2}{3} \mu\left(\nabla \cdot \mathrm{U}_{\mathrm{p}}\right) \mathbf{I}-2 \frac{\mu \kappa_{\mathrm{p}}}{p} \nabla(\nabla p)+\frac{\rho \kappa_{\mathrm{p}}}{p} \mathrm{U}_{\mathrm{p}} \otimes \nabla p+\frac{\rho \kappa_{\mathrm{p}}}{p} \nabla p \otimes \mathrm{U}_{\mathrm{p}}
\end{aligned}
$$

Here, $\mathrm{D}\left(\mathrm{U}_{\mathrm{p}}\right)$ denotes the symmetric part of the velocity gradient. A parallel can be drawn between the structure of tensor $\mathbf{T}^{(R N S)}$ and Korteweg's stress tensor $\mathbf{T}$ [22]. Korteweg augmented the Newtonian stress tensor with the dyadic product $\nabla \rho \otimes \nabla \rho$ to represent forces experienced by fluids during phase transitions. His complete tensor may be written as in [23]:

$$
\mathbf{T}=\left(-p+\alpha_{0}|\nabla \rho|^{2}+\alpha_{1} \nabla^{2} \rho\right) \mathbf{I}+\beta \nabla \rho \otimes \nabla \rho+2 \mu \mathrm{D}(\mathbf{v})-\lambda(\nabla \cdot \mathbf{v}) \mathbf{I},
$$

where the material coefficients $\alpha_{0}, \alpha_{1}, \beta, \mu, \lambda$ may depend on $\rho$ as well. On comparing eqn (9) with eqn (8), it is seen that all terms involved in the structure of the Korteweg stress tensor are found in the recast Navier-Stokes tensor, but written with $p$ rather than $\rho$.

\section{THE NEW OPENFOAM IMPLEMENTATION}

The standard installation of OpenFOAM [24] comes with at least five incompressible solvers most of which are based on the PISO algorithm. In the incompressible Navier-Stokes equations, mass balance appears as a kinematic constraint on the velocity field (eqn (1)). There is no independent equation for the pressure, which presents a problem for the numerical computation of the solution. Solvers of the PISO family address this issue by constructing a Poisson equation for the pressure to enforce mass conservation, using one or more correction loops at each time-step [25]. This makes the solution of pressure expensive.

In the Recast Navier-Stokes setting, the pressure appears explicitly in the mass balance equation (eqn (6)). Instead of modifying one of the existing incompressible solvers, a simple sequential algorithm is proposed. This is shown in the source code of the new solver rnsLiquidFlow in Listing 1 below. The following steps may be identified here:

1. Define and compute the new diffusive term Jp and the surface flux of Jp (lines 1-3).

2. Define the part of the momentum equation involving the velocity Up (lines 6-12). 
3. Solve the above momentum equation for velocity field using the pressure field from the previous time step (lines 14-19).

4. Define equation for pressure field (lines 21-24).

5. Solve for the pressure field using the previously calculated solution for velocity field (line 26).

6. Repeat the steps $1-5$ for next time step.

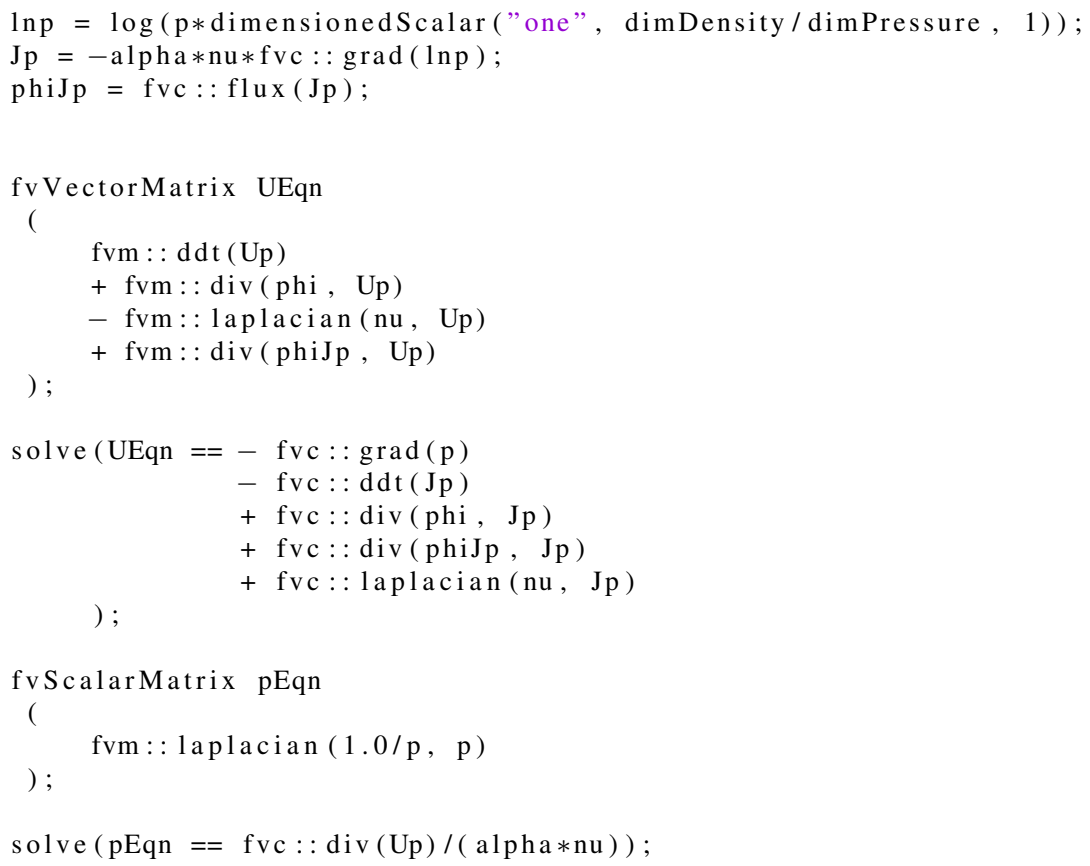

Listing 1: Excerpt of source code in rnsLiquidFoam.C

\section{CREEPING FLOW IN CYLINDRICAL NANO-CHANNELS}

\subsection{Perturbation solutions}

The tube-like channels embedded in a CNT membrane are characterised by a very small diameter $D$ compared to their length $L$. It is therefore reasonable to seek a perturbation solution of eqns (6)-(7) in the channel aspect ratio $\varepsilon=D / L$, employing a cylindrical polar coordinate system $(r, \theta, z)$ in which the components of the pressure velocity are denoted $\mathrm{U}_{\mathrm{p}_{\mathrm{r}}}$, $\mathrm{U}_{\mathrm{p}_{\theta}}$ and $\mathrm{U}_{\mathrm{p}_{\mathrm{z}}}$. Fig. 1 shows a schematic of the fluid flow,

which is assumed to be axisymmetric and with $\mathrm{U}_{\mathrm{p}_{\theta}}=0$. The flow is driven by a pressure field $p$, such that $p(r, 0)=\mathrm{P}_{\text {in }}$ and $p(r, L)=\mathrm{P}_{\text {out }}$ for all $0 \leq r \leq R$, where $\mathrm{P}_{\text {in }}>\mathrm{P}_{\text {out }}$ are the constant inlet and outlet pressures. On the channel wall, the tangential component of the velocity is assumed to satisfy the no-slip condition, i.e. $\mathrm{U}_{\mathrm{p}_{\mathrm{z}}}(R, z)=0$, so that any mass flow tangent to the wall must be purely diffusive. The radial component $\mathrm{U}_{\mathrm{p}_{\mathrm{z}}}(R, z)$ on the other hand is related to the diffusive mechanism in such a way that no mass passes through the wall:

$$
\mathrm{U}_{\mathrm{m}_{\mathrm{r}}}(R, z)=\mathrm{U}_{\mathrm{p}_{\mathrm{r}}}(R, z)-\left.\kappa_{\mathrm{p}} \frac{\partial \ln p}{\partial r}\right|_{(R, z)}=0 .
$$




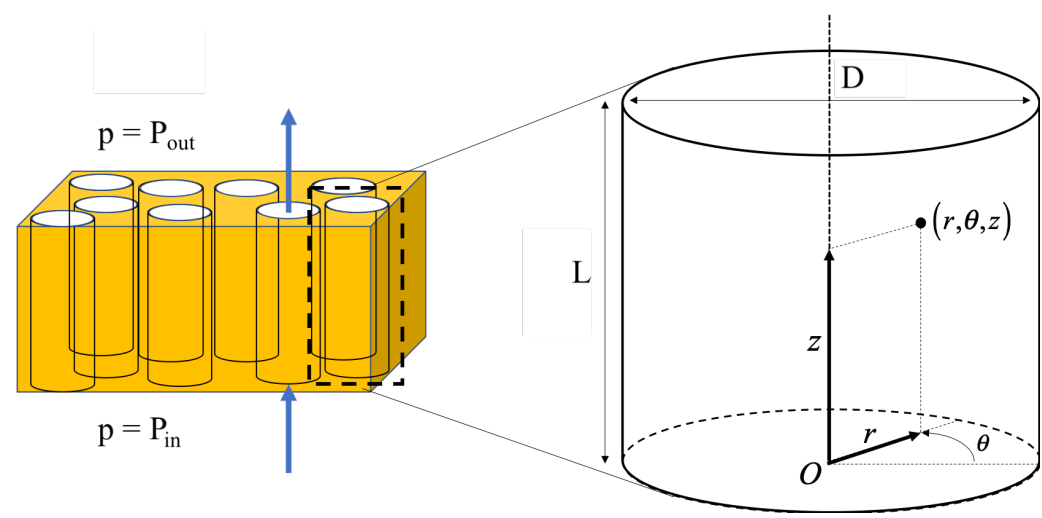

Figure 1: Schematic of CNT membrane and cylindrical geometry.

Eqn (10) suggests that some arbitrariness is left unless the gradient of the pressure at the wall is specified and this will be commented on in the solver validation section below.

In Table 1, dimensionless variables are defined in which the suggested perturbation solution is written as:

$$
\begin{gathered}
\tilde{p}(\tilde{r}, \tilde{z})=\tilde{p}_{0}(\tilde{r}, \tilde{z})+\varepsilon \tilde{p}_{1}(\tilde{r}, \tilde{z})+\varepsilon^{2} \tilde{p}_{2}(\tilde{r}, \tilde{z})+\cdots, \\
\tilde{\mathrm{U}}_{\mathrm{p}_{\mathrm{r}}}(\tilde{r}, \tilde{z})=\tilde{\mathrm{U}}_{\mathrm{p}_{\mathrm{r}}, 0}(\tilde{r}, \tilde{z})+\varepsilon \tilde{\mathrm{U}}_{\mathrm{p}_{\mathrm{r}}, 1}(\tilde{r}, \tilde{z})+\varepsilon^{2} \tilde{\mathrm{U}}_{\mathrm{p}_{\mathrm{r}}, 2}(\tilde{r}, \tilde{z})+\cdots, \\
\tilde{\mathrm{U}}_{\mathrm{p}_{\mathrm{z}}}(\tilde{r}, \tilde{z})=\tilde{\mathrm{U}}_{\mathrm{p}_{\mathrm{z}}, 0}(\tilde{r}, \tilde{z})+\varepsilon \tilde{\mathrm{U}}_{\mathrm{p}_{\mathrm{z}}, 1}(\tilde{r}, \tilde{z})+\varepsilon^{2} \tilde{\mathrm{U}}_{\mathrm{p}_{\mathrm{z}}, 2}(\tilde{r}, \tilde{z})+\cdots .
\end{gathered}
$$

For the dimensional analysis of different flow regimes the characteristic velocity magnitude $|\mathrm{U}|_{c}$ is related to the characteristic pressure $P_{c}$ via:

$$
|\mathrm{U}|_{c}=\frac{P_{c} D^{2}}{\mu L}=\frac{P_{c} \varepsilon H}{\mu} .
$$

The Reynolds' number $\mathrm{Re}_{\mathrm{p}}$ is calculated with respect to the pressure velocity $\mathrm{U}_{\mathrm{p}}$ and can now be expressed as:

$$
\operatorname{Re}_{\mathrm{p}}=\frac{|\mathrm{U}|_{c} \rho D}{\mu}=\frac{P_{c} \rho \varepsilon D^{2}}{\mu^{2}} .
$$

A Nusselt number $\mathrm{Nu}$ may be defined to compare the diffusive and advective transport mechanisms. With $\kappa_{p}$ defined by eqn (5), this is directly related to the Reynolds' number:

$$
\mathrm{Nu}=\frac{\kappa_{\mathrm{p}}}{U H}=\frac{\alpha^{*}}{\operatorname{Re}_{\mathrm{p}}} .
$$

Table 1: Definition of dimensionless variables.

\begin{tabular}{|l||c|c|c|c|c|}
\hline Dimensional variable & $\mathrm{z}$ & $\mathrm{r}$ & $\mathrm{U}_{\mathrm{p}, \mathrm{z}}$ & $\mathrm{U}_{\mathrm{p}, \mathrm{r}}$ & $\mathrm{p}$ \\
\hline Scaling factor & $\mathrm{L}$ & $\mathrm{D}$ & $|U|_{c}$ & $|U|_{c}$ & $P_{c}$ \\
\hline Dimensionless variable & $\tilde{z}=\frac{z}{L}$ & $\tilde{r}=\frac{r}{D}$ & $\tilde{\mathrm{U}}_{p, z}=\frac{\mathrm{U}_{\mathrm{p}, \mathrm{z}}}{|U|_{c}}$ & $\tilde{\mathrm{U}}_{p, r}=\frac{\mathrm{U}_{\mathrm{p}, \mathrm{r}}}{|U|_{c}}$ & $\tilde{p}=\frac{p}{P_{c}}$ \\
\hline
\end{tabular}


4.2 Flow regime with $\operatorname{Re}_{\mathrm{p}}=O\left(\varepsilon^{2}\right)$ and $\alpha^{*}=O(1)$

Table 2 in Section 5 lists a set of parameters typical for flow experiments carried out in nano-diameter channels of small aspect ratio. Based on these values, the dimensionless numbers $\varepsilon=1 \times 10^{-3}$ and $\operatorname{Re}_{\mathrm{p}}=1 \times 10^{-5}$ are found. The low Reynolds' number is typical of creeping flows so that inertial terms can be neglected in the momentum equation. A rigorous perturbation analysis on the non-dimensionalised equations with $\operatorname{Re}_{\mathrm{p}}=O\left(\varepsilon^{2}\right)$ and $\alpha^{*}=O(1)$ reveals that the first two pressure terms in eqn (11) are independent of the radial coordinate $r$ (see [16] for details). In other words, $\tilde{p}_{0}=\tilde{p}_{0}(z)$ and $\tilde{p}_{1}=\tilde{p}_{1}(z)$. Using this and equating the $\mathrm{O}(1)$ terms in the mass balance equation results in:

$$
\tilde{p}_{0}^{2} \frac{1}{\tilde{r}} \frac{\partial\left(\tilde{r} \tilde{\mathrm{U}}_{\mathrm{p}_{\mathrm{r}}, 0}\right)}{\partial \tilde{r}}=\frac{\alpha^{*}}{\operatorname{Re}_{\mathrm{p}}}\left[\varepsilon^{2} \tilde{p}_{0} \frac{d^{2} p_{0}}{d \tilde{z}^{2}}-\varepsilon^{2}\left(\frac{d \tilde{p}_{0}}{d \tilde{z}}\right)^{2}+\tilde{p}_{0} \frac{1}{\tilde{r}} \frac{\partial}{\partial \tilde{r}}\left(\tilde{r} \frac{\partial \tilde{p}_{2}}{\partial \tilde{r}}\right)\right] .
$$

It should be noted here that, within the perturbation analysis, the $\mathrm{O}(1)$ term of the radial component of the mass velocity (see eqn (10)) is:

$$
\tilde{p}_{0} \tilde{\mathrm{U}}_{\mathrm{m}_{\mathrm{r}}, 0}=\tilde{p}_{0} \tilde{\mathrm{U}}_{\mathrm{pr}_{\mathrm{r}}, 0}-\frac{\alpha^{*}}{\operatorname{Re}_{\mathrm{p}}} \frac{\partial \tilde{p}_{2}}{\partial \tilde{r}}
$$

The pressure term $\tilde{p}_{0}(\tilde{z})$ can be found directly by integrating eqn (17) and using the boundary condition $\tilde{\mathrm{U}}_{\mathrm{m}_{\mathrm{r}}, 0}=0$ at the wall. This leads to the following ordinary differential equation:

$$
\tilde{p}_{0} \frac{d^{2} \tilde{p}_{0}}{d \tilde{z}^{2}}-\left(\frac{d \tilde{p}_{0}}{d \tilde{z}}\right)^{2}=0
$$

Introducing the ratio $\mathcal{P}=\mathrm{P}_{\text {in }} / \mathrm{P}_{\text {out }}$, the dimensional form of the solution is:

$$
p_{0}(z)=\mathrm{P}_{\text {in }} \exp \left(-\frac{\ln (\mathcal{P}) z}{L}\right) \Longleftrightarrow \frac{z}{L} \ln \mathcal{P}+\ln \frac{p_{0}(z)}{\mathrm{P}_{\text {in }}}=0
$$

Furthermore, equating the $\mathrm{O}(1)$ terms in the $z$-momentum equation yields:

$$
\frac{\partial^{2} \tilde{\mathrm{U}}_{\mathrm{p}_{\mathrm{z}}, 0}}{\partial \tilde{r}^{2}}+\frac{1}{\tilde{r}} \frac{\partial \tilde{\mathrm{U}}_{\mathrm{p}_{\mathrm{z}}, 0}}{\partial \tilde{r}}=\frac{d \tilde{p}_{0}}{d \tilde{z}} .
$$

With application of the no-slip condition on $\tilde{U}_{\mathrm{p}_{z}, 0}$, eqn (21) results in a parabolic velocity profile, the dimensional form of which is:

$$
\mathrm{U}_{\mathrm{p}_{\mathrm{z}}, 0}(r, z)=\frac{1}{4 \mu}\left(r^{2}-R^{2}\right) \frac{\mathrm{d} p_{0}}{\mathrm{~d} z}
$$

It should be emphasized that, in the high Nusselt number flow regime considered here, the stream-wise velocity term $U_{p_{z}, 0}$ does not feature in eqn (17). The diffusive transport dominates and determines the pressure distribution with help of the no-penetration condition at the wall. 
4.3 Flow regime with $\operatorname{Re}_{\mathrm{p}}=O\left(\varepsilon^{2}\right)$ and $\alpha^{*}=O(\varepsilon)$

In Stamatiou et al. [16], the case with $\operatorname{Re}_{\mathrm{p}}=O(\varepsilon)$ and $\alpha^{*}=O(1)$ was investigated. However, closer inspection reveals that the same terms dominate the equations for the flow regime in which $\operatorname{Re}_{\mathrm{p}}=O\left(\varepsilon^{2}\right)$ and $\alpha^{*}=O(\varepsilon)$. This is because the Nusselt number $\mathrm{Nu}=\alpha^{*} / \operatorname{Re}_{\mathrm{p}}$ is the same for both these regimes and the terms proportional to the Reynolds number are negligible. In comparison to the case examined in Section 4.2, there is one significant change: while the $z$-momentum equation is again given by eqn (21), the mass balance equation now exhibits an extra term coupling the two equations together:

$$
\tilde{p}_{0}^{2} \frac{\partial \tilde{\mathrm{U}}_{\mathrm{p}_{\mathrm{z}}, 0}}{\partial \tilde{z}}+\tilde{p}_{0}^{2} \frac{1}{\tilde{r}} \frac{\partial\left(\tilde{r} \tilde{\mathrm{U}}_{\mathrm{p}_{\mathrm{r}}, 0}\right)}{\partial \tilde{r}}=\frac{\alpha^{*}}{\operatorname{Re}_{\mathrm{p}}}\left[\varepsilon^{2} \tilde{p}_{0} \frac{d^{2} p_{0}}{d \tilde{z}^{2}}-\varepsilon^{2}\left(\frac{d \tilde{p}_{0}}{d \tilde{z}}\right)^{2}+\tilde{p}_{0} \frac{1}{\tilde{r}} \frac{\partial}{\partial \tilde{r}}\left(\tilde{r} \frac{\partial \tilde{p}_{2}}{\partial \tilde{r}}\right)\right] .
$$

The pressure term $\tilde{p}_{0}(\tilde{z})$ is now found by substituting the dimensionless form of eqn (22) in eqn (23), integrating and using $\tilde{\mathrm{U}}_{\mathrm{m}_{\mathrm{r}}, 0}=0$ at the wall. The resulting ordinary differential equation for $\tilde{p}_{0}(\tilde{z})$ now becomes:

$$
\frac{\mathrm{d}^{2} \tilde{p}_{0}}{\mathrm{~d} \tilde{z}^{2}}+\frac{32 \alpha^{*} \varepsilon}{\operatorname{Re}_{\mathrm{p}}} \frac{\mathrm{d}^{2} \ln \tilde{p}_{0}}{\mathrm{~d} \tilde{z}^{2}}=0 .
$$

This equation only admits an implicit solution, the dimensional form of which can be written as follows:

$$
p_{0}(z)+\frac{8 \mu \kappa_{\mathrm{p}}}{R^{2}}\left[\frac{z}{L} \ln \mathcal{P}+\ln \frac{p_{0}(z)}{\mathrm{P}_{\mathrm{in}}}\right]=\mathrm{P}_{\text {in }}+\frac{\Delta P}{L} z,
$$

where $\mathcal{P}=\mathrm{P}_{\text {in }} / \mathrm{P}_{\text {out }}$ as before and $\Delta P=\mathrm{P}_{\text {out }}-\mathrm{P}_{\text {in }}$ is the pressure drop. Written in this form, it can be seen that the pressure distribution for the present flow regime is a correction of eqn (20) by a linear pressure drop. Eqn (20) is approximated for large values of $\mu \kappa_{\mathrm{p}} / R^{2}$, while the Haagen-Poiseuille flow law is recovered for small values of $\mu \kappa_{\mathrm{p}} / R^{2}$. This is also seen when considering the mass flow rate expression:

$$
\dot{\mathrm{M}}_{\mathrm{RNS}}=\frac{\pi \rho R^{4}}{8 \mu L}\left[\Delta P+\frac{8 \mu \kappa_{\mathrm{p}}}{R^{2}} \ln (\mathcal{P})\right]=\dot{\mathrm{M}}_{\mathrm{HP}}\left(1+E_{\mathrm{RNS}}\right),
$$

where $\dot{\mathrm{M}}_{\mathrm{HP}}$ is the no-slip Haagen-Poiseuille mass flow rate, i.e.

$$
\dot{\mathrm{M}}_{\mathrm{HP}}=\frac{\pi \rho R^{4} \Delta P}{8 \mu L},
$$

and $E_{\mathrm{RNS}}$ is the flow enhancement factor defined as,

$$
E_{\mathrm{RNS}}=\frac{\dot{\mathrm{M}}_{\mathrm{RNS}}-\dot{\mathrm{M}}_{\mathrm{HP}}}{\dot{\mathrm{M}}_{\mathrm{HP}}}=\frac{8 \mu \kappa_{\mathrm{p}} \ln (\mathcal{P})}{R^{2} \Delta P} \text {. }
$$

\section{SOLVER VALIDATION}

\subsection{Computational mesh and solver settings}

In this section it is verified that the rnsLiquidFoam solver agrees with the previously found analytical expressions for flows in cylindrical tubes. The axial symmetry of the problem 
permits the solution of the equations on a small wedge instead of the entire cylinder. Fig. 2 shows the computational mesh for a wedge angle of $5^{\circ}$ with 100 uniformly spaced cells in the z-direction and 20 cells in the y-direction with an expansion ratio of 0.1 , so that the mesh becomes more refined in the near-wall region. The small angle allows the wall to be approximated by a flat boundary patch with outward normal $\hat{n}$ in the $y$-direction. On this boundary, the conditions $\mathrm{U}_{\mathrm{p}}=(0,0,0)$ and $\nabla p \cdot \hat{n}=0$ (zeroGradient) are adopted, which is stricter than the boundary condition used to derive the first order perturbation solutions in Section 4 (see eqn (10)). The angled top and bottom planes of the mesh are equipped with the wedge condition for rotationally symmetric cases. Table 2 summarises the other physical parameters used for the simulation of water flow in a CNT of $10 \mathrm{~nm}$ diameter and $10 \mu \mathrm{m}$ length driven by a pressure difference of 1 bar.

\subsection{Comparison with analytical solutions}

In Section 4.3 the influence of the diffusivity parameter $\alpha^{*}$ on the pressure distribution was highlighted. This is illustrated in Fig. 3, where eqn (25) is plotted for $\alpha^{*}=1$ and $\alpha^{*}=0.001$, representing extremal values for a large range of possible diffusivities $\kappa_{\mathrm{p}}$. The same curves are also computed with rnsLiquidFoam, sampling along the $z$-axis. Excellent agreement can be seen in both cases. On the one hand, the case with $\alpha^{*}=1$ corresponds to a flow process dominated by the diffusive transport mechanism, the convective transport being negligible. On the other hand, the case with $\alpha^{*}=0.001$ leads to both mechanisms contributing equally. As $\alpha$ decreases, the pressure distribution is seen to approach the linear pressure drop of classical Poiseuille flow. For illustrative purposes, the value $\alpha^{*}=0.005$ is used in all subsequent plots, which represents a case where diffusive transport is of the same

Table 2: Parameters for simulation of water flow in CNTs.

\begin{tabular}{|l|c|c|c|c|c|c|}
\hline Parameter & $\mathrm{L}(\mathrm{m})$ & $\mathrm{D}(\mathrm{m})$ & $\mu\left(\mathrm{kg} \mathrm{m}^{-1} \mathrm{~s}^{-1}\right)$ & $\rho\left(\mathrm{kg} \mathrm{m}^{-3}\right)$ & $\mathrm{P}_{\text {in }}(\mathrm{Pa})$ & $\mathrm{P}_{\text {out }}(\mathrm{Pa})$ \\
\hline Value & $1 \times 10^{-5}$ & $1 \times 10^{-8}$ & $1 \times 10^{-3}$ & $1 \times 10^{3}$ & $2 \times 10^{5}$ & $1 \times 10^{5}$ \\
\hline
\end{tabular}

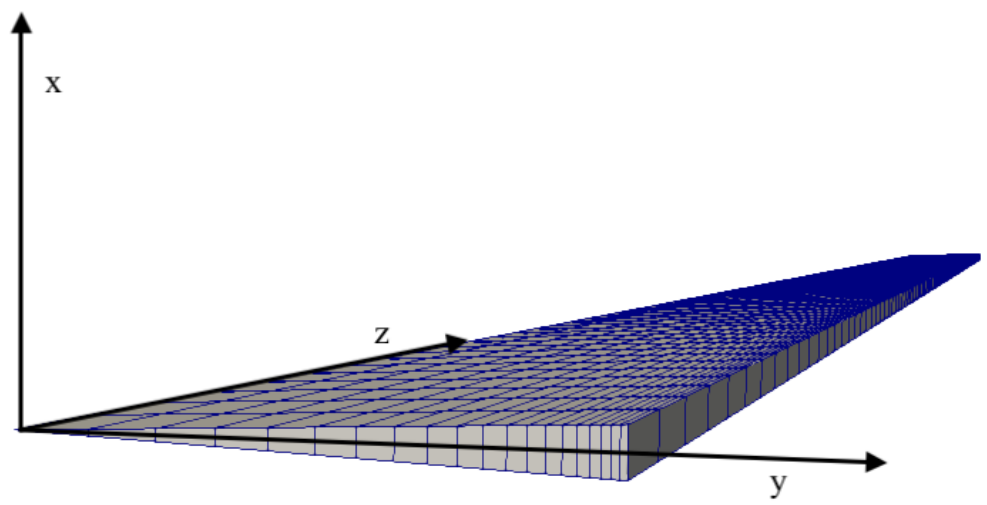

Figure 2: Computational mesh for a wedge of $5^{\circ}$. 


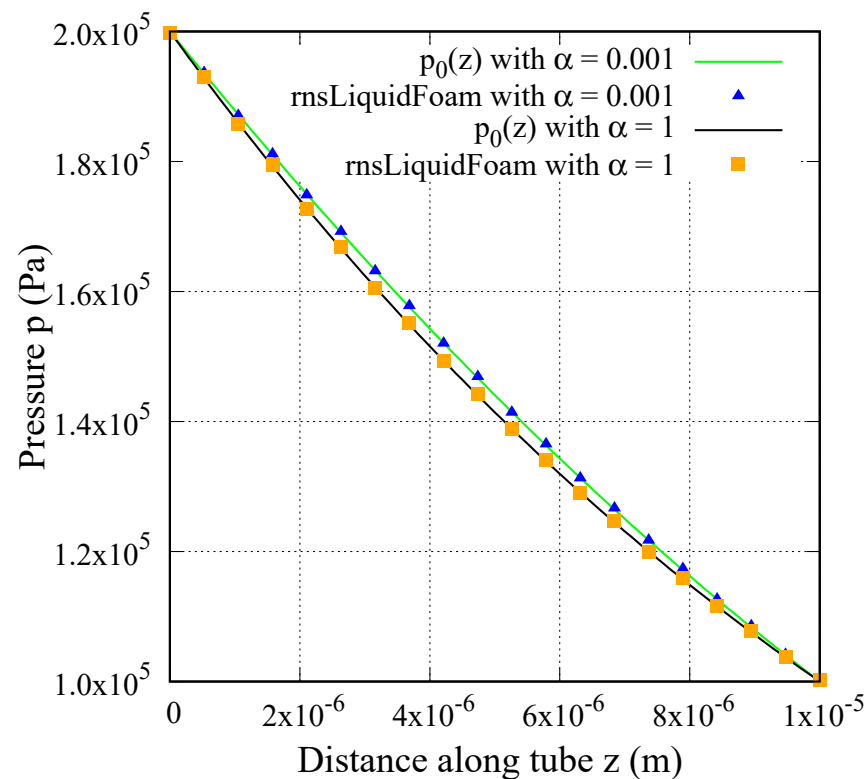

Figure 3: Pressure distribution along the tube.

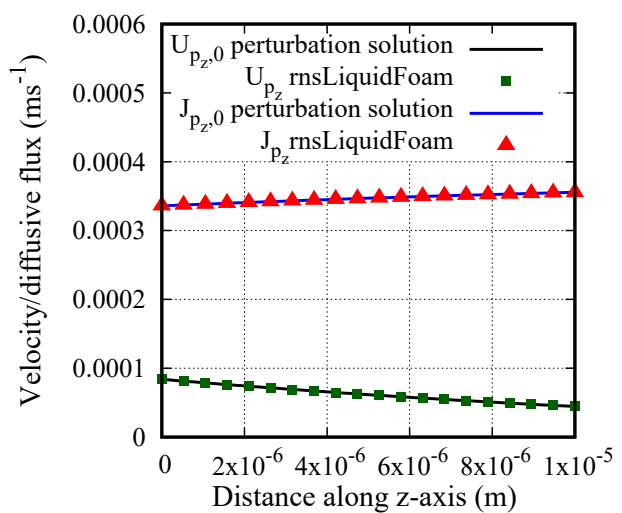

(a)

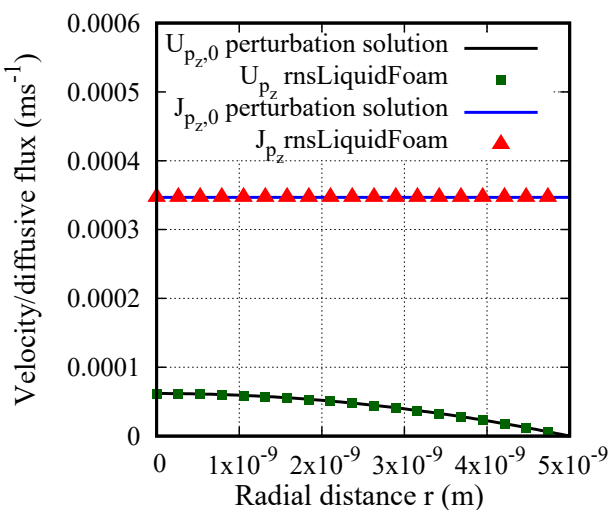

(b)

Figure 4: Stream-wise velocity and diffusion components on two axes.

order but clearly higher than convective transport. Fig. 4(a) shows the variation of streamwise components $\mathrm{U}_{\mathrm{p}_{\mathrm{z}}}$ and $\mathrm{J}_{\mathrm{p}_{z}}$ on the $z$-axis. 


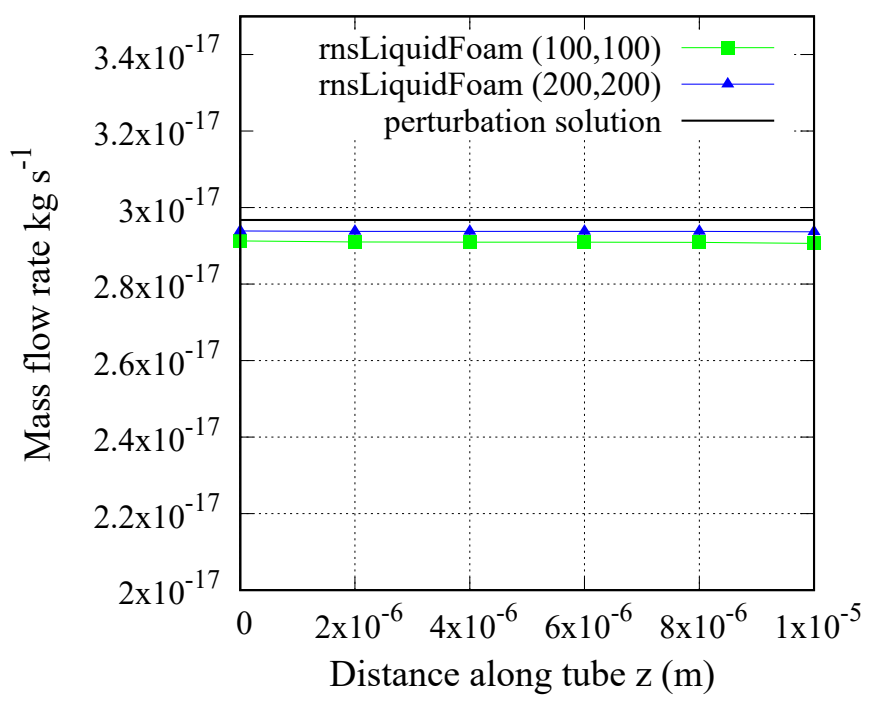

Figure 5: Mass flow rate calculation on two grids.

Both are non-constant, owing to the choice of $\alpha^{*}$ (not too 'small' or 'large'). Fig. 4(b) shows the same components plotted against the radial distance from the $z$-axis at the centre of the channel $\left(z=5 \times 10^{-9} \mathrm{~m}\right)$. The numerical solution of $\mathrm{U}_{\mathrm{p}_{z}}$ agrees well with the parabolic profile $\mathrm{U}_{\mathrm{p}_{\mathrm{z}, 0}}$ (eqn (22)) and $\mathrm{J}_{\mathrm{p}_{\mathrm{z}}}$ is constant. The mass velocity profile $\mathrm{U}_{\mathrm{m}_{\mathrm{z}}}$ is therefore also parabolic and indistinguishable from that obtained using a velocity slip condition in the classical Navier-Stokes equations.

Fig. 5 shows the calculation of the mass flow rate through six representative cross sections of the entire tube. This was initially done using a $100 \times 100$ grid (green squares) and then repeated on a $200 \times 200$ (blue triangles) refined grid. The mass flow rate is constant along the channel in both numerical calculations and the solution appears to converge to eqn (26). A deviation with respect to this analytical expression must persist also because the circular tube wall has been approximated by a polygonal wall.

Fig. 6 shows the flow enhancement for a selection of experimental studies and molecular dynamics simulations of water flow in CNTs, which differ both in channel dimensions and in imposed pressure differences. Eqn (28) is plotted (orange triangles connected by straight line segments) using the dimensions and pressures quoted in each individual investigation. A geometry-dependent form of $\alpha^{*}$ has been employed here, given by $\alpha^{*}=0.003 \times L / D$ [16]. The corresponding simulation results from the new solver, rnsLiquidFoam, are shown by the blue open circles connected with dashed straight line segments.

It should be noted that molecular dynamics simulations have revealed that the radial pressure distribution in nano-sized tubes is not constant, dropping off sharply near the boundaries [10]. Similarly, the viscosity is slightly reduced in this zone, which has been incorporated in previous modelling attempts [15]. The kinematic viscosity $\nu=\mu / \rho$ enters as 


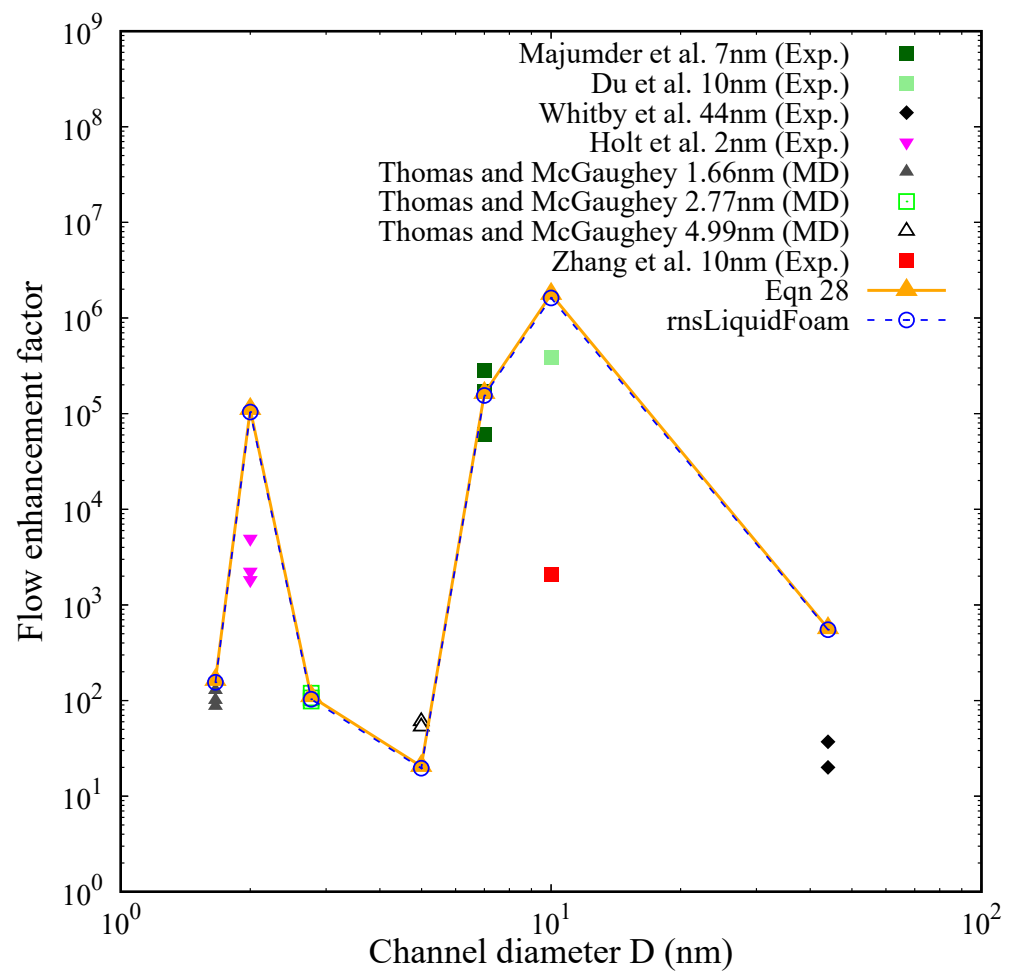

Figure 6: Comparison between numerically simulated, experimental and analytical flow rate enhancement.

a constant in the new model and considering its radial variation could lead to an improved agreement with the data. Also the inclusion of end-effects should be examined, as suggested in Sisan and Lichter [11].

\section{CONCLUSIONS}

This paper has explored the numerical implementation in OpenFOAM of a Recast NavierStokes equations for pressure-driven liquid flows. The explicit appearance of the pressure in the mass balance equation suggests a simplified numerical solution process compared to that of the typical incompressible Navier-Stokes equations where mass balance must be enforced by a Poisson equation for the pressure and several correction steps. For liquid flow in CNTs, perturbation solutions in the small channel aspect ratio were reviewed and used to test convergence of the new solver. Very good agreement was obtained for the pressure distribution and velocity profiles. The calculation of the mass flow rate showed a small deviation from the analytical expression, but appeared to converge to it upon grid-refinement. We also showed that the solver computes the correct mass flow rates for the different channel sizes and pressure differences used in experimental and molecular dynamics studies. 


\section{ACKNOWLEDGEMENTS}

This research is supported by the UK's Engineering and Physical Sciences Research Council (EPSRC) under grant no. EP/R008027/1 and The Leverhulme Trust, UK, under grant Ref. RPG-2018-174.

\section{REFERENCES}

[1] Majumder, M., Chopra, N., Andrews, R. \& Hinds B.J., Enhanced flow in carbon nanotubes. Nature, 438, p. 44, 2005.

[2] Holt, J.K., Park, H.G., Wang, Y., Stadermann, M., Artyukhin, A.B., Grigoropoulos, C.P., Noy, A. \& Bakajin, O., Fast mass transport through sub2-nanometer carbon nanotubes. Science, 312, pp. 1034-1037, 2006.

[3] Majumder, M., Chopra, N., Andrews, R. \& Hinds, B.J., Mass transport through carbon nanotube membranes in three different regimes: Ionic diffusion and gas and liquid flow. Nano, 5, pp. 3867-3877, 2011.

[4] Qin, X., Yuan, Q., Zhao, Y., Xie, S. \& Liu, Z., Measurement of the rate of water translocation through carbon nanotubes. Nano Lett., 11, pp. 2173-2177, 2011.

[5] Du, F., Qu, L., Xia, Z., Feng, L. \& Dai, L., Membranes of vertically aligned superlong carbon nanotubes. Langmuir, 27, pp. 8437-8443, 2011.

[6] Whitby, M., Cagnon, L., Thanou, M. \& Quirke, N., Enhanced fluid flow through nanoscale carbon pipes. Nano Lett., 8, pp. 2632-2637, 2008.

[7] Sinha, S., Rossi, M. P., Mattia, D. \& Godotsi, Y., Induction and measurement of minute flow rates through nanopipes. Phys. Fluids, 19, 013603, 2007.

[8] Joseph, S. \& Aluru, N.R., Why are carbon nanotubes fast transporters of water? Nano Lett., 8, pp. 452-458, 2008.

[9] Thomas, J.A. \& McGaughey, A.J.H., Reassessing fast water transport through carbon nanotubes. Nano Lett., 8, pp. 2788-93, 2008.

[10] Walther, J.H., Ritos, K., Cruz-Chu, E.R., Megaridis, C.M. \& Koumoutsakos, P., Barriers to superfast water transport in carbon nanotube membranes. Nano Lett., 13, pp. 19101914, 2013.

[11] Sisan, T.B. \& Lichter, S., The end of nanochannels. Microfluid Nanofluid, 11, pp. 787$791,2011$.

[12] Kannam, S.K., Todd, B.D., Hansen, J.S. \& Daivis, P.J., How fast does water flow in carbon nanotubes? J. Chem. Phys., 138, 094701, 2013.

[13] Hansen, J.S., Todd, B.D. \& Daivis, P.J., Prediction of fluid velocity slip at solid surfaces. Phys. Rev. E, 84, 016313, 2011.

[14] Kannam, S.K., Todd, B.D., Hansen, J.S. \& Daivis, P.J., Interfacial slip friction at a fluidsolid cylindrical boundary. J. Chem. Phys., 136, 244704, 2012.

[15] Myers, T.G., Why are slip lengths so large in carbon nanotubes? Microfluidics and Nanofluidics, 10(5), pp. 1141-1145, 2010.

[16] Stamatiou, A., Dadzie, S.K. \& Reddy, M.H.L., Investigating enhanced mass flow rates in pressure-driven liquid flows in nanotubes. J. Phys. Commun., 3, p. 125012, 2019.

[17] Reddy, M.H.L., Dadzie, S.K., Ocone, R., Borg, M.K. \& Reese, J.M., Recasting NavierStokes equations. J. Phys. Commun., 3, p. 105009, 2019.

[18] Dadzie, S.K. \& Reddy, M.H.L., Recasting Navier-Stokes equations: Shock wave structure description. AIP Conference Proceedings, 2293, p. 050005, 2020.

[19] Dadzie, S.K., Reese, J.M. \& McInnes, C.R., A continuum model of gas flows with localized density variations. Physica A, 387, pp. 6079-6094, 2008.

[20] Brenner, H., Beyond Navier-Stokes. Int. J. Eng. Sci., 54, pp. 67-98, 2012. 
[21] Dadzie, S. K. \& Brenner, H., Predicting enhanced mass flow rates in gas microchannels using nonkinetic models. Phys. Rev. E, 86, 2012.

[22] Korteweg, D.J., Sur la forme que prennent les equations du mouvements des fluides si l'on tient compte des forces capillaires causees par des variations de densite considerables mais connues et sur la theorie de la capillarite dans l'hypothese d'une variation continue de la densite. Arch. Neerl. Sci. Ex. Nat. (ii) 6, 1901.

[23] Heida, M. \& Malek, J., On compressible Korteweg fluid-like materials. Int. J. Eng. Sci., special Issue in Honor of K.R. Rajagopal., 48(11), pp. 1313-1324, 2010.

[24] Weller, H.G., Tabor, G., Jasak, H. \& Fureby, C., A tensorial approach to computational continuum mechanics using object-oriented techniques. Computers in Physics, 12, p. 620, 1998.

[25] Ferziger, J.H. \& Peric, M., Computational Methods for Fluid Dynamics. SpringerVerlag: Berlin, Heidelberg, New York, 2002. 